\title{
New Phylosophy for pole amplitude modulation in the three phase induction machines.
}

\author{
F.E.Abdel-kader A.E.Abou Mobarka W.S.Abouel-Fadl. \\ Dept. of Elect. Eng., Faculty of Eng. \\ Minufiya University, Shebien El-Kom, Egypt.
}

\section{Abstract}

The induction motor is considered to be one of the best electrical motors However up to now, it is difficult to control its speed by an ideal method, or even by a method close to that of direct current motors. In spite of this disadvantage, this motor is widely used when the load is not in need of control of its speed over a wide range. Some loads need to change the speed by a ratio of two or three times the rated value. In this case, it is possible to use the Pole Amplitude Modulation to control the method of speed . This method does not need high cost and keeps the motor characteristics in a high grade. The previous papers on this subject mentioned that this technique can be carried out by different methods of modulating the connections of motor winding parts. There is a contradiction between theory and practice seemed when verifying these connections experimentally on an actual motor, so that the predicted speeds could not be obtained. So the motor performance has been analyzed mathematically and physically. From these results, the conditions necessary for motor windings, their distribution and the method of connection have been obtained.

Manuscript received from Eng; W. S.Abouel - Fadl on : $16 / 6 / 1999$

Accepted on: 12/9/1999

Engineering Research Bulletin, Vol 22,No 3, 1999

Minufiya University, Faculty of Engineering, Shebin El-Kom , Egypt, ISSN 1110-1180 


\section{1-Introduction}

Induction motors are inherently single speed devices and some form of control over the speed has been the subject of the academic pursuit almost since the induction motor was invented. One of these forms is the pole amplitude modulation technique. This can be done in several methods, for example :

a) Series parallel switching.

b) Reversal of a complete half winding such as :

1- Parallel star / series delta.

2. Parallel star/series star.

There is a contradiction between theoretical analysis and practical application seemed when applying these methods experimentally on these motors.

A tested motor is wound as a double layer, 4 pole 60 degree phase spread. Each phase consists of four groups connected in series. Conventional operation of this motor is to run at 1500 r.p.m. When the different techniques of pole amplitude madulation discussed in the previous papers are applied to give 2- poles, 3000 r.p.m., the motor failed to rotate. The probabilities of this problem are investigated in this paper.

\section{2- Probabilities of desired speed failure :}

The probabilities of the desired speed failure have been studied as follows:

\section{2-1 Rotor number of slots :}

It is known that with the number of stator slots $S_{1}$ equals to the number of rotor slots $S_{2}$, the machine may refuse to start at all (cogging). But in this motor $S_{1}=36$ slots while $S_{2}=28$ slots.

In certain values of rotor number of slots, the motor may exhibit a tendency to run at low speeds (crawling ). The causes of crawling may be :

\section{a-Asynchronous harmonic torques :}

To limit these harmonic torques $S_{2}$ should not exceed $1.25 S_{1}$. In the tested motor $S_{2}$ is less than $S_{1}$ ( 28 slots ).

\section{b-Synchronous torques :}

To redues these torques

$S_{2} \neq 6 \mathrm{px}$ or $6 \mathrm{px} \neq 2 \mathrm{p}^{(1)}$ where $\mathrm{x}$ is an integer.

In our case for $x=1$ and $2 p=2$

$S_{2}=28 \neq 6$ px 
also $6 \mathrm{px} \neq 2 \mathrm{p}$

c-Slot harmonics.

To mitigate the slot harmonics:

$S_{2} \neq S_{1} \pm 2 p$ or $S_{1} \pm p$ or $1 / 2 S_{1} \pm p$

In the tested motor

$S_{2} \neq 36 \pm 2,(38$ or 34$)$ or $(36 \pm 1)$ or $(36 / 2 \pm 1)$

Although the previous conditions are satisfied, the motor still could not run by the high speed (3000 r.p.m.).

\section{2-2- Rotor skewing :}

To avoid the effect of unacurate rotor skewing and rotor number of slots on the slot harmonics, a solid rotor has been used instead of the squirrel cage rotor. The motor still in the crawling case and could not run by the high speed (3000 r.p.m) .

This means that the number of rotor slots or their skewing are not responsible for this deffect.

\section{2-3 Winding current waveform :}

The waveforms of stator current at no load for different connections are recorded by the storage oscilloscope and drawn by the $x-y$ recorder as shown in Fig (1). It is noticed that there is a distortion in the waveforms, which indicates that the mmf and thus the air gap flux may be the reason of failure of obtaining the required speed.

To show the reasons of this distortion in the magnetic field, the effect of wounding the coils from the point of phase spread and the number of layers has been studied as follows:

\section{2-4 Phase spread of the winding :}

The effect of phase spread of the winding has been studied theoretically and experimentally for the following cases :

1- Single layer , $4 \& 2$ poles .

2- Double layer, 60 degrees phase spread for $4 \& 2$ poles .

3- Double layer, 120 degrees phase spread for $4 \& 2$ poles.

The corresponding winding diagrams are indicated in Figure (2) (a,b,c,d,e \& f) respectively.

\section{2-4-1 Single layer winding}

\section{(a) 4-Pole connection}

Figure $(2 \mathrm{a}, \mathrm{b})$ shows the connection diagram of a single layer winding for 4 pole connection and 2-pole connection.

Figure (3) shows the distribution of ampere conductors (AC) in each slot at values of $w t=0,15,30, \ldots \& 345^{\circ}$ respectively. All these figures show a 4pole waveform for time instants.

It can be noted that the field is rotating, and so it gives the predicted speed (1500 r.p.m.) . This is the ideal field for the 4 - pole connection . 


\section{(b) 2-pole connection}

Figure (4) shows the distribution of ampere conductors (AC) in each slot at values of $w t=0,15, \ldots \& 345^{\circ}$ respectively, for 2-pole connection.

It is stationary for $80^{\circ}$ from group no. 3 to group no. 10 then it moves to start from group 2 to group 9 . For $60^{\circ}$. Suddenly it starts from group no. 10 leaving 4 groups at $w t=160^{\circ} \& 180^{\circ}$. It moves smoothly again. From these figures, it may be concluded that the rotor refuses to rotate at the predicted speed (3000 r.p.m.) . The field is distorted and pulsating instead of rotating.

\section{2-4-2 Double layer winding with $60^{\circ}$ phase spread}

\section{(a) 4-pole connection}

The motor under test is connected to give $2 \mathrm{p}=4$. The number of slots / pole $/$ phase $=3$.

Figure (5) shows the $\mathrm{AC}$ distribution in each slot, at values of $\mathrm{wt}=0,15,30$, ... $345^{\circ}$ respectively. Each 3 slots are named in group numbers $1,2, \ldots . \& 12$. It may be noted that at different values of wt, the 4-poles are obtained with equal areas. From these figures it may be noted that the field rotates, so it gives the predicted speed (1500 r.p.m.).

\section{(b) 2-pole connection}

The current in half of the winding is reversed to obtain the 2-pole operation. Fiigure (6) shows the distribution of $\mathrm{AC}$ at each slot at $\mathrm{wt}=0,15$, ... \& $345^{\circ}$ respectively.

From these figures it may be noted that the field also pulsates instead of rotates, so that the rotor refuses to rotate at the predicted speed (i.e. 3000 r.p.m.).

\section{2-4-3 Double layer winding with 120 phase spread :}

The tested motor is reconnected to give $120^{\circ}$ phase spread as shown in Fig. ( 2 e,f), to give $4 \& 2$ poles respectively.

(a) 4 - pole connection :

Figure (7) shows the Ac distribution in each slot at values of $w t=0$, $15,30 \ldots . . \& 345^{\circ}$ respectively.

It may be noted that at different values of wt, the 4-poles are obtained with equal areas. Also the field rotates, so it gives the 1500 r.p.m.

\section{(b) 2-pole connection :}

The motor could run at 3000 r.p.m. which is the predicted speed .

Figure (8) shows the Ac distribution of this connection at $w t=0,15,30, \&$ $345^{\circ}$ respectively.

It may be concluded that in the case of 2-pole $120^{\circ}$ phase spread, the field rotates which enables the rotor to move with 3000 r.p.m., while in the case of $60^{\circ}$ phase spread the field pulsates, so that the rotor fails to rotate with 3000 r.p.m. 


\section{3- Experimental Results :}

A motor of $120^{\circ}$ phase spread is tested at both speeds (1500. \& 3000 r.p.m.s ) as follows :

\section{3-1 No- load test}

Figures $(9,10 \& 11)$ show the no load power, input current and power factor respectively against the supply voltage .

\section{3-2 Load Test}

The motor is loaded by another induction motor when the stator windings are excited by direct currents. Figures. $(12,13,14 \& 15)$ show the variation of motor speed, power factor, efficiency, and input power against the output torque. All motor characteristics are higher at 3000 r.p.m. compared with that at 1500 r.p.m., also the efficiency and the power factor are better at 3000 r.p.m.

\section{4- Conclusion}

Induction motors using the pole amplitude modulation technique should have the following conditions to enable the rotor to rotate with the predicted speeds. (a) The well known conditions for all induction motors :

$1-S_{2} \neq S_{1}$ to avoid cogging .

2- $\mathrm{S}_{2}$ should not exceed $1.25 \mathrm{~S}_{1}$ to avoid harmonic induction torques.

3- $S_{2} \neq 6 \mathrm{px}$ or $6 \mathrm{px} \neq 2 \mathrm{p}$ where $\mathrm{x}=1,2, .$. to avoid harmonic synchronous torques.

4- $S_{2} \neq S_{1} \pm 2 p$

$\neq S_{1} \pm p$

$\neq 1 / 2 S_{1} \pm p$

to avoid the slot harmonics.

(b) The conditions for pole amplitude modulation :

1- The winding must be double layer $120^{\circ}$ phase spread.

2- The motor characteristics will be improved at high speed rather than at low speed, when the number of turns per phase is equal at both speeds.

\section{References}

(1) Fong, W., and Rawcliffe, G.H. : "Three speed single - winding squirrelcage induction motors", Proc. IEE, 1963, 110, (9), pp. 1649 - 1655.

(2) A.R.W. Broadway, and J.L. Rodrignez : "Delta - star PAM", Proc. IEE, $1981,128,(5)$, pp. $255-250$. 
(3) Fong, W. : Wide - ratio two - speed single - winding induction motors", ibid., 1965, 112, (7), pp. 1335 - 1347.

(4) Rajaraman, K.C. : " New 2-speed induction motors", Proc. IEE, 1978 , 125, (1), pp. 957 - 958.

(5) Rawacliffe, G. H., and Fong, W. : " Close - ratio two - speed single winding induction motors", ibid., 1963, 110, (5) , p. 916.

(6) Rajaraman, K.C. , Ranjith, W. and Subramaniam, G. : New wide - ratio pole - changing motors", Proc. IEE, 1987, 134 (4) , pp. 211 - 214.

(7) The Performance And Design of Alternating Current Machines . M.G. SAY.

\section{Appendix}

\section{Data of the tested motor.}

The data of the 3-phase induction motor used in the laboratory measurements are; $0.75 \mathrm{HP}, 380 / 220$ - V, 1.6 / $2.77 \mathrm{~A}, 50 \mathrm{HZ}, 1425$ r.p.m., 0.71 effeciency, 0.74 power factor, 36 stator slots, 28 rotor slots.

The motor parameters are ;

Stator phase resistance $=14.2 \Omega$

Stator phase leakage reactance $=25 \Omega$

Stator phase magnetising reactance $=250 \Omega$

Equivalent iron loss phase resistance $=2900 \Omega$ 


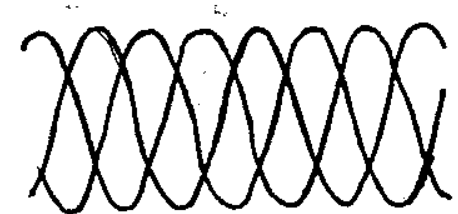

(a)

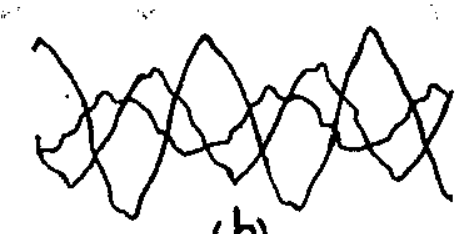

(b)

Fig.(1)

(a) MMF waveform for 4-pole connection.

(b) MMF waveform for 2-pole connection.

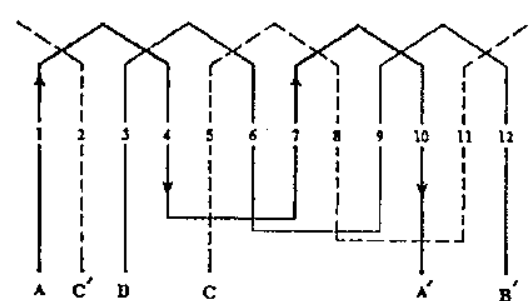

(a) Single layer, 60 degrees, 4 poles.

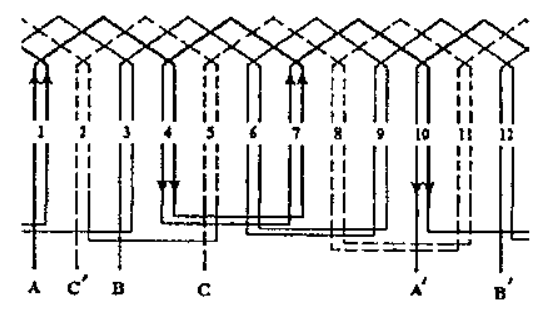

(c) Double layer, 60 degrees, 4 poles

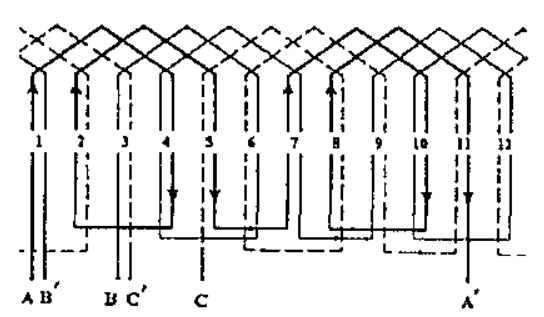

(e) Double layer, 120 degrees, 4 poles.

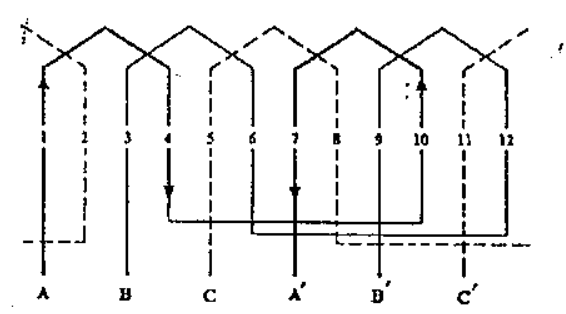

(b) Single layer, 60 degrees, 2 poles .

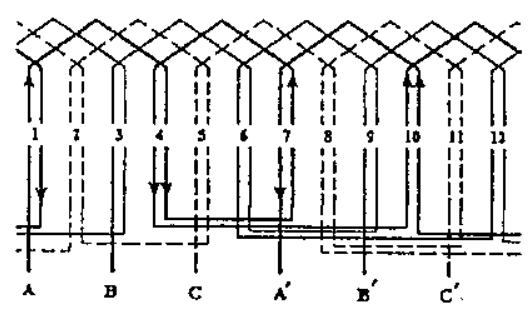

(d) Double layer, 60 degrees, 2 poles .

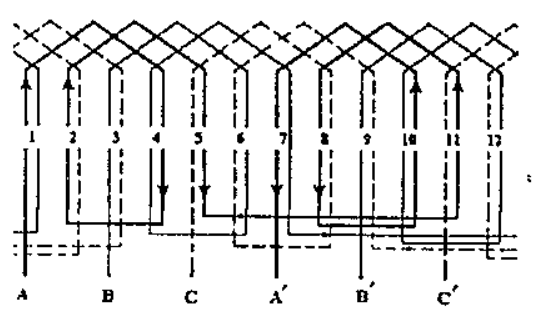

(f) Dinuble layer, 120 degrees, 2 poles.

Fig.(2).Winding diagram for :

1- Single layer $60^{\circ}$ phase spread (a,b).

2-Double layer $60^{\circ}$ phase spread (c,d).

3-Double layer $120^{\circ}$ phase spread $(e, f)$. 

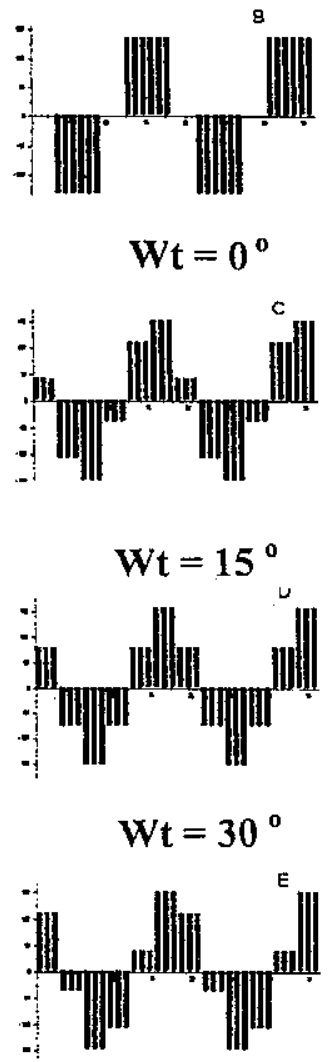

$$
W t=45^{\circ}
$$

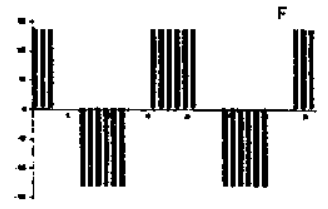

$$
\mathrm{Wt}=60^{\circ}
$$

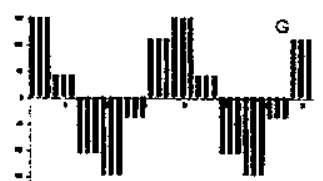

$W t=75^{\circ}$

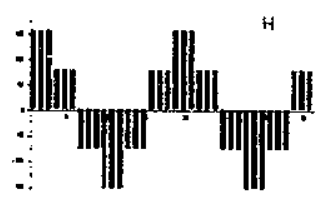

$$
W t=90^{\circ}
$$

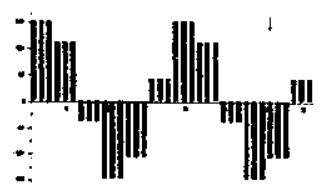

$W t=105^{\circ}$

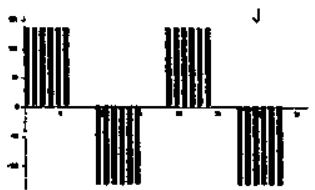

$\mathrm{Wt}=120^{\circ}$

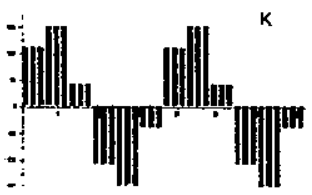

$W t=135^{\circ}$

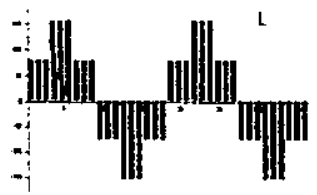

$\mathrm{Wt}=150^{\circ}$

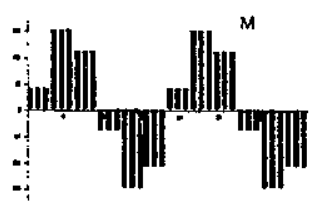

$\mathrm{Wt}=165^{\circ}$

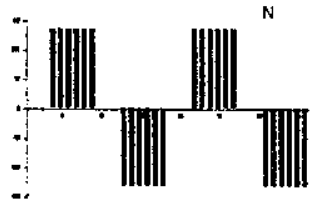

$\mathrm{Wt}=180^{\circ}$

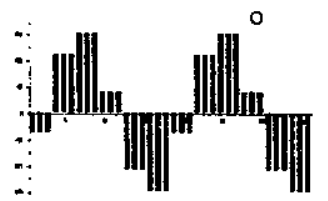

$W t=195^{\circ}$

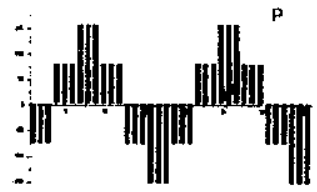

$\mathrm{Wt}=210^{\circ}$

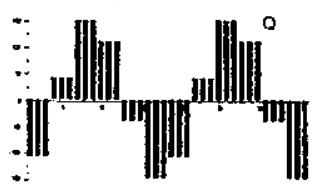

$W t=225^{\circ}$
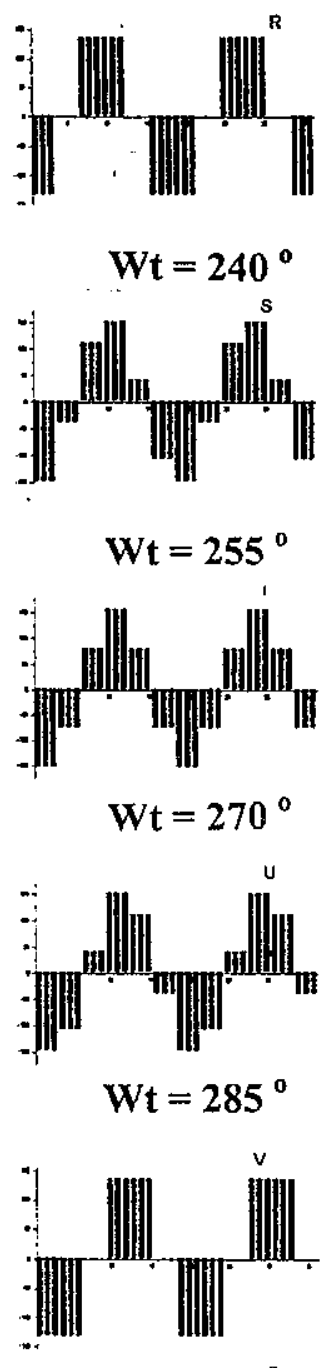

$W t=300^{\circ}$

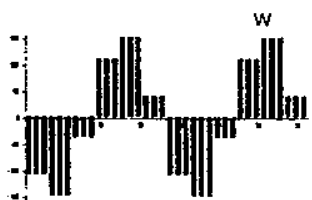

$\mathrm{Wt}=315^{\circ}$
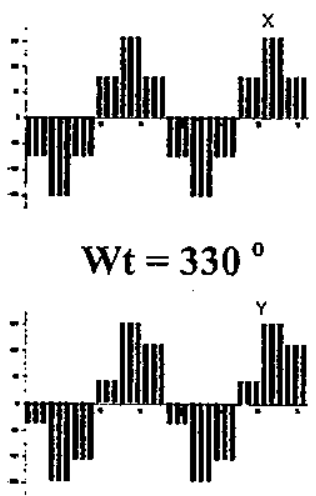

$\mathrm{Wt}=345^{\circ}$

Fig .(3). AC distribution of single layer winding for 4-pole connection at $\mathrm{Wt}=0,15,30, \ldots . \& 345^{\circ}$. 

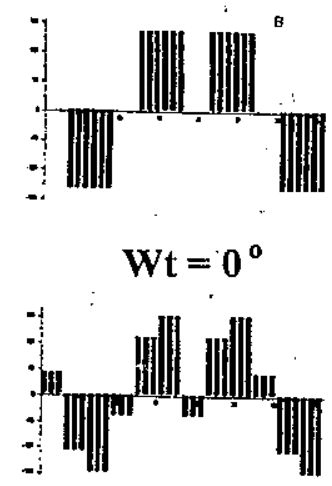

$$
\mathrm{Wt}=15^{\circ}
$$

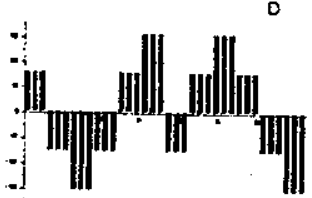

$$
W t=30^{\circ}
$$

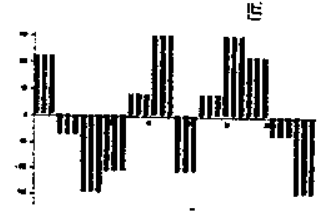

$W t=45^{\circ}$

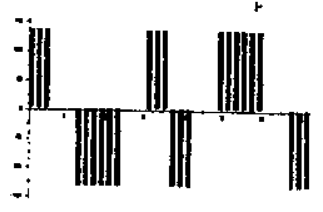

$$
\mathrm{Wt}=60^{\circ}
$$

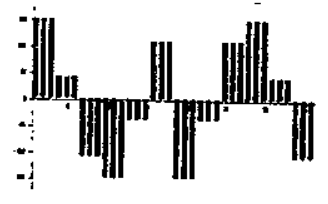

$$
\mathrm{Wt}=75^{\circ}
$$

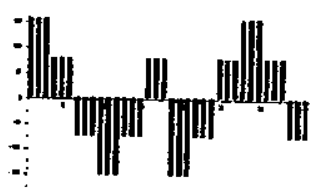

$$
\mathrm{Wt}=90^{\circ}
$$

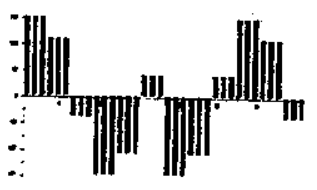

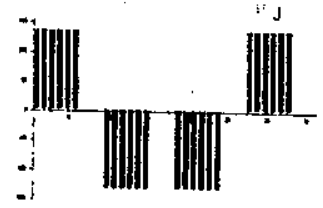

$\mathrm{Wt}=120^{\circ}$

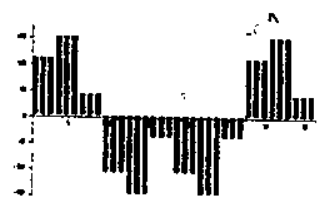

$W t=135^{\circ}$
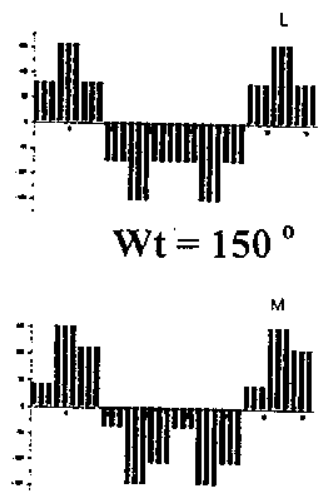

$W t=165^{\circ}$
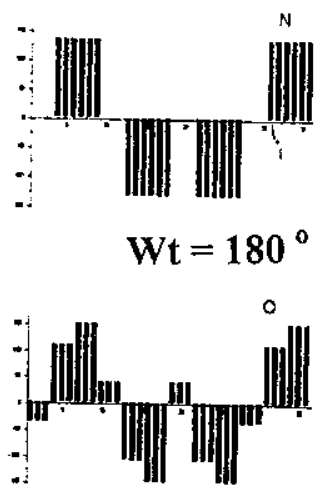

$\mathrm{Wt}=195^{\circ}$

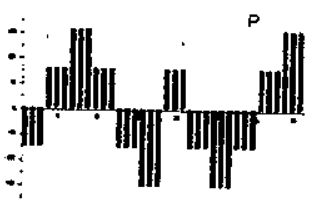

$$
\mathrm{Wt}=210^{\circ}
$$

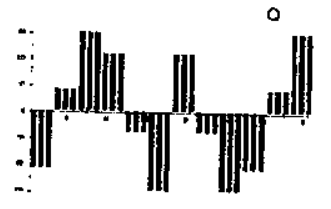

$$
W t=105^{\circ}
$$

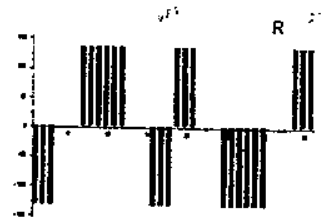

$\mathrm{Wt}=240^{\circ}$

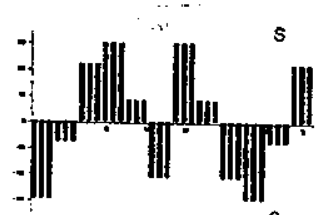

$\mathrm{Wt}=255^{\circ}$

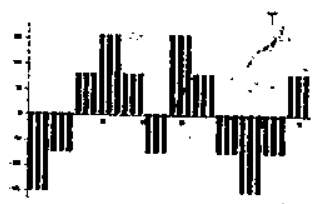

$\mathrm{Wt}=270^{\circ}$

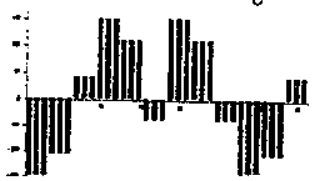

$\mathrm{Wt}=285^{\circ}$
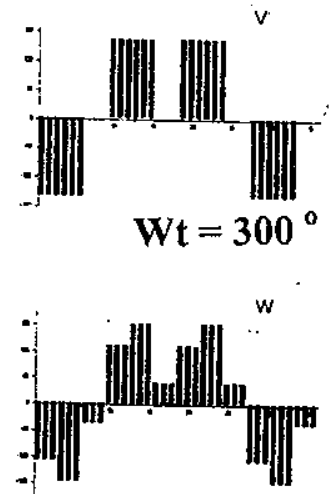

$\mathrm{Wt}=315^{\circ}$

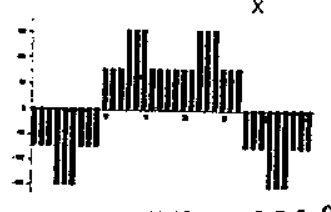

$\mathrm{Wt}=330^{\circ}$

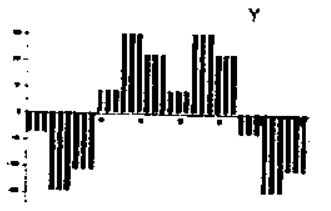

$W t=345^{\circ}$

Fig. (4). AC distribution of single layer winding for 2-pole connection at $\mathrm{W}_{\mathrm{t}}=0,15,30, \ldots \& 345^{\circ}$. 

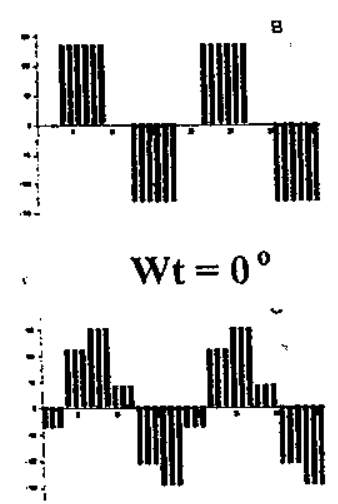

$$
\mathrm{Wt}=15^{\circ}
$$
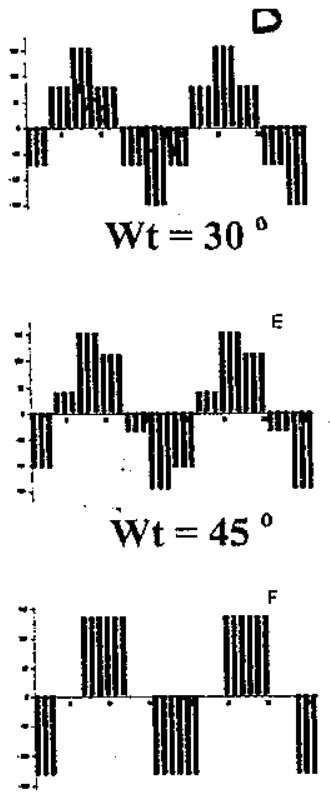

$$
\mathrm{Wt}=60^{\circ}
$$
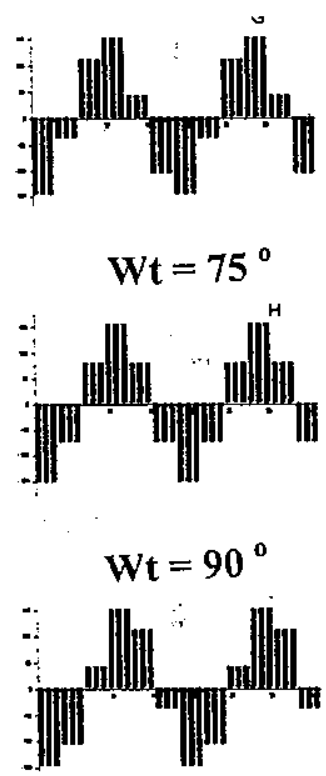

$$
W_{t}=105^{\circ}
$$
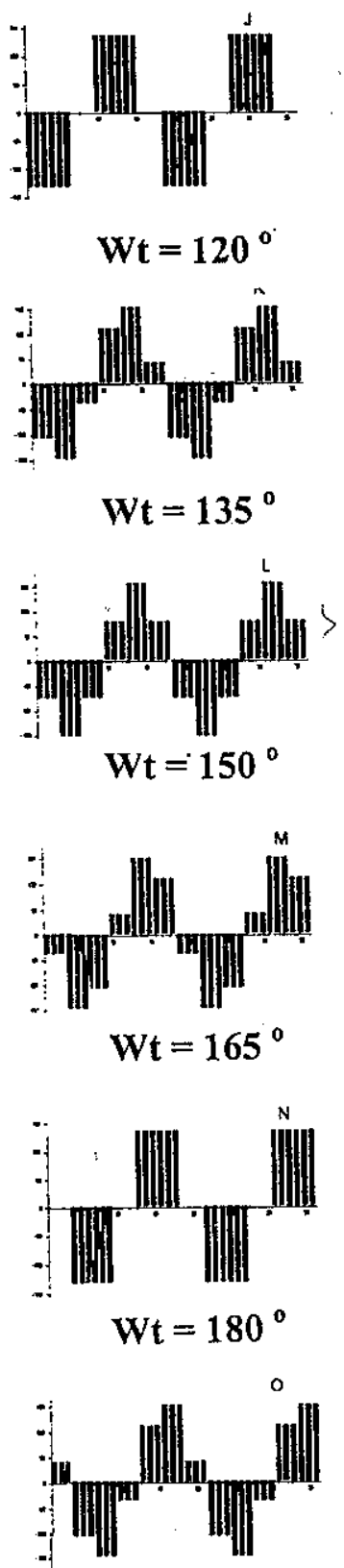

$$
W t=195^{\circ}
$$

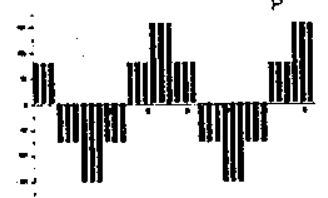

$$
\mathrm{Wt}=210^{\circ}
$$

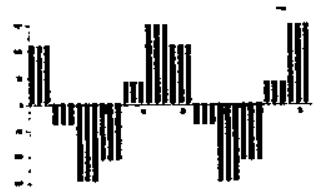

$$
W t=225^{\circ}
$$

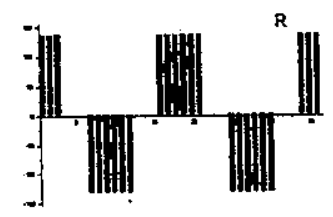

$\mathrm{Wt}=\mathbf{2 4 0 ^ { \circ }}$

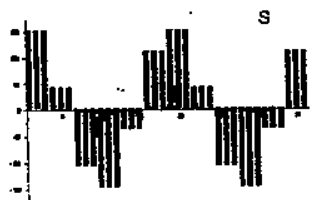

$$
W t=255^{\circ}
$$
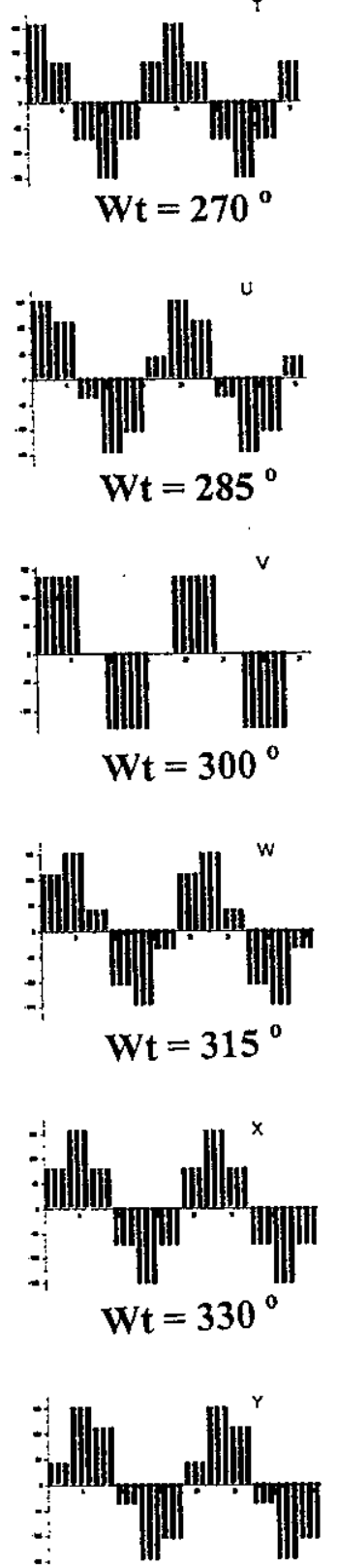

$\mathrm{Wt}=345^{\circ}$

Fig.(5). AC distribution of double layer winding $60^{\circ}$ phase spread for 4-pole connection at wt $=0,15,30, \ldots 345^{\circ}$. 


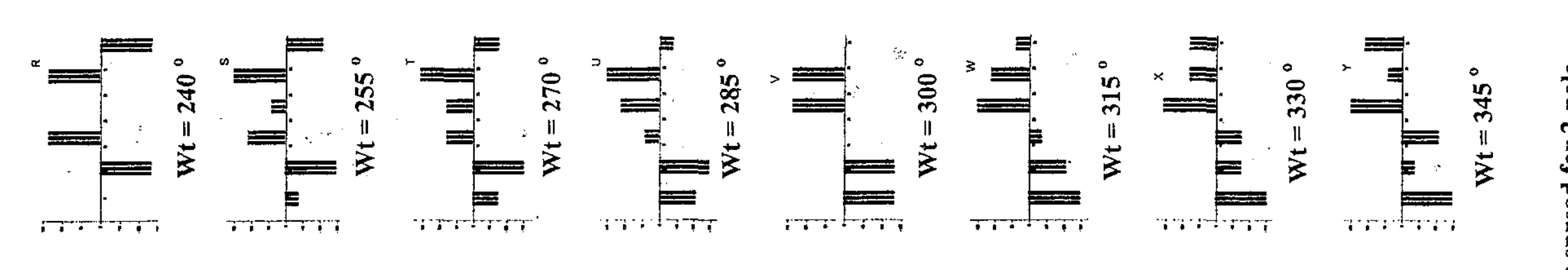

$$
==0
$$



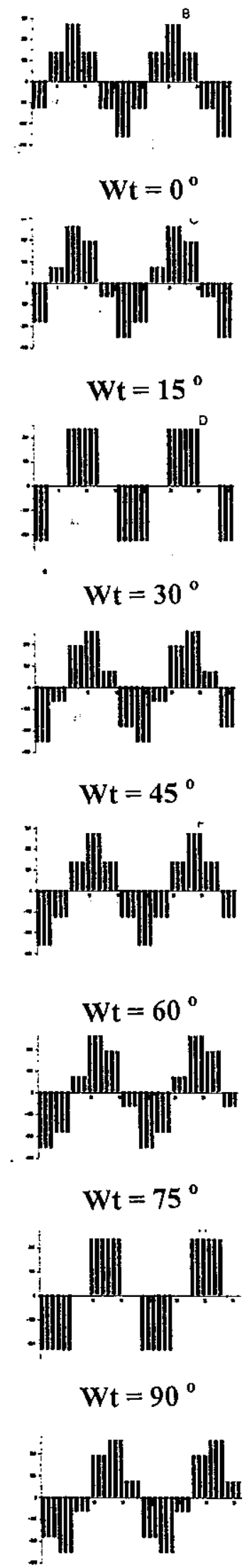

$$
\mathrm{Wt}=105^{\circ}
$$

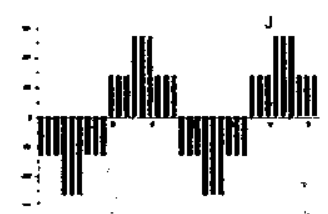

$$
\mathrm{Wt}=120^{\circ}
$$

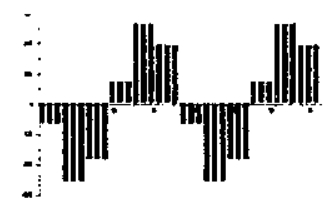

$$
\mathrm{Wt}=135^{\circ}
$$

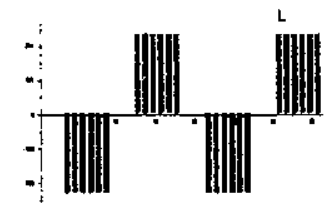

$$
\mathrm{Wt}=150^{\circ}
$$

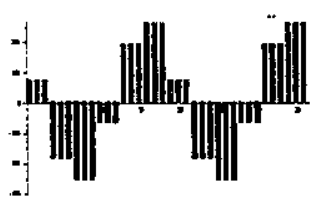

$$
\mathrm{Wt}=165^{\circ}
$$

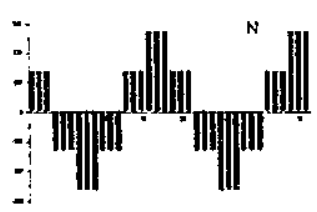

$$
\mathrm{Wt}=180^{\circ}
$$

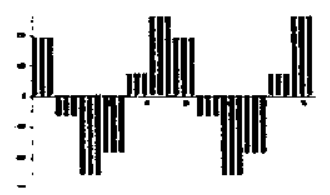

$$
W t=195^{\circ}
$$

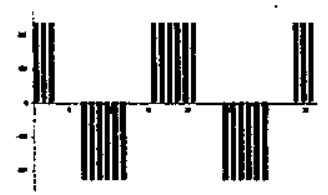

$$
\mathrm{Wt}=210^{\circ}
$$

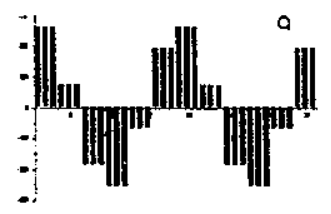

$$
W t=225^{\circ}
$$

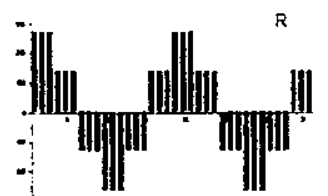

$\mathrm{Wt}=240^{\circ}$

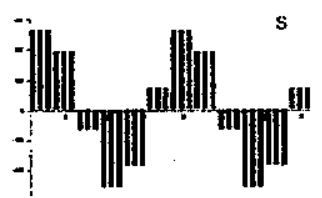

$\mathrm{Wt}=255^{\circ}$
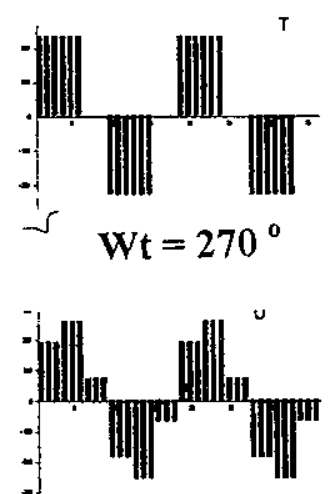

$$
W t=285^{\circ}
$$

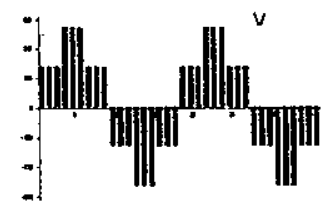

$$
\mathrm{Wt}=300^{\circ}
$$

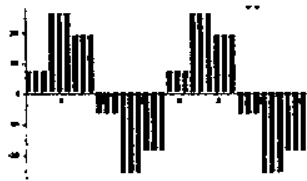

$$
W_{t}=315^{\circ}
$$

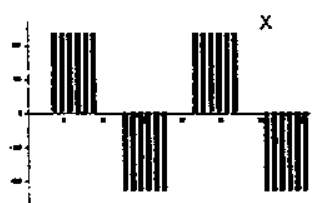

$$
\mathrm{Wt}=330^{\circ}
$$

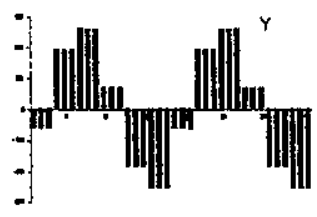

$$
\mathrm{Wt}=345^{\circ}
$$

Fig.(7). AC distribution of double layer winding $120^{\circ}$.phase spread for 4-pole connection at wt $=0,15,30, \ldots 345^{\circ}$. 

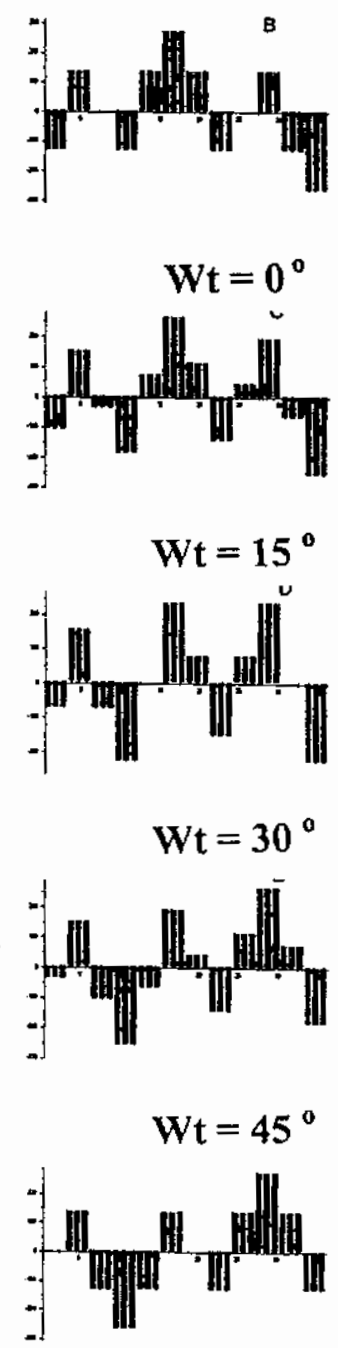

$$
\mathrm{Wt}=60^{\circ}
$$

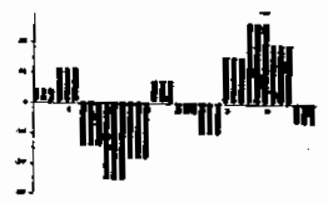

$$
W \mathrm{t}=75^{\circ}
$$

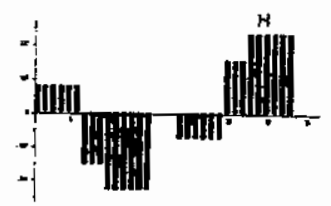

$$
W t=90^{\circ}
$$

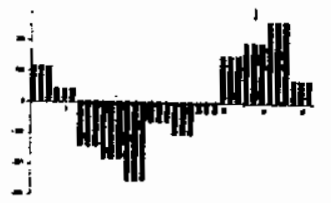

$$
W t=105^{\circ}
$$

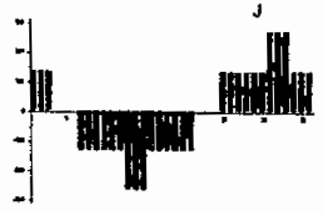

$\mathbf{W t}=120^{\circ}$

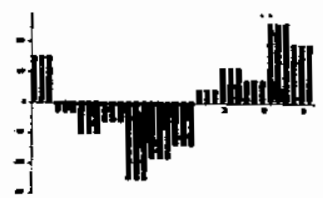

$W t=135^{\circ}$

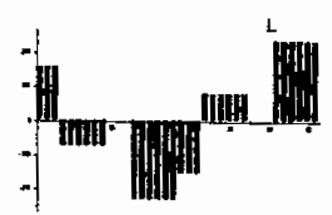

$\mathrm{Wt}=150^{\circ}$

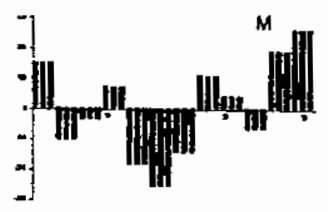

$W t=165^{\circ}$

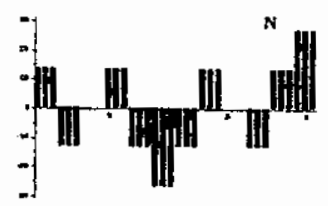

$\mathrm{Wt}=180^{\circ}$

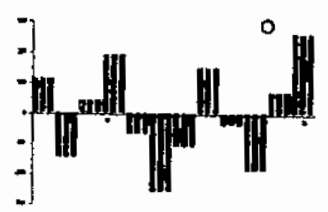

$$
W t=195^{\circ}
$$

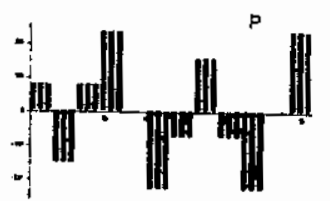

$$
W t=210^{\circ}
$$

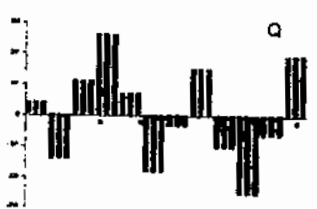

$W t=225^{\circ}$
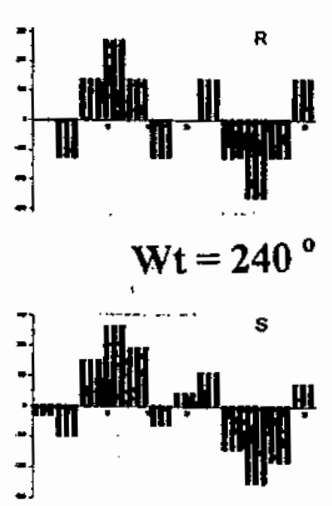

$\mathrm{Wt}=255^{\circ}$

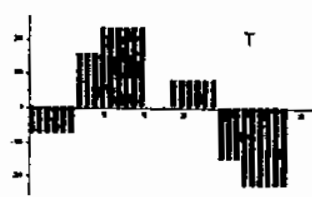

$W t=270^{\circ}$

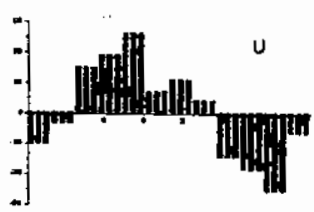

$\mathrm{Wt}=285^{\circ}$

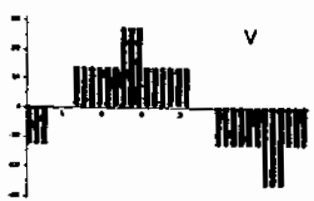

$\mathrm{Wt}=300^{\circ}$

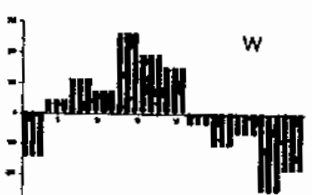

$W t=315^{\circ}$

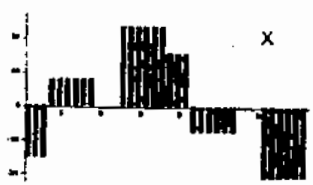

$\mathrm{Wt}=330^{\circ}$

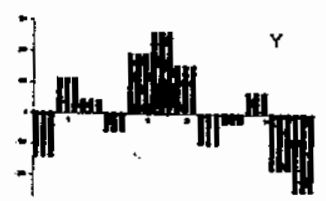

$W t=345^{\circ}$

Fig.(8).AC distribution of double layer winding $120^{\circ}$ phase spread for 2-pole connection at $w t=0,15,30, \ldots 345^{\circ}$. 


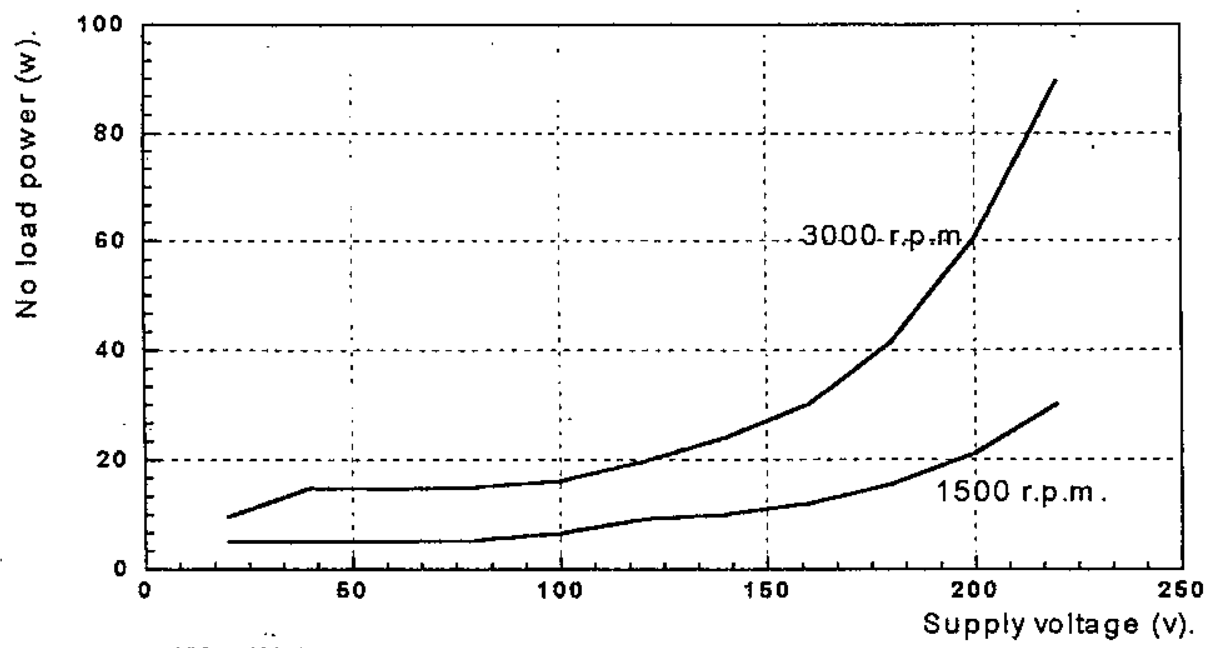

Fig.(9).No load power versus supply voltage.

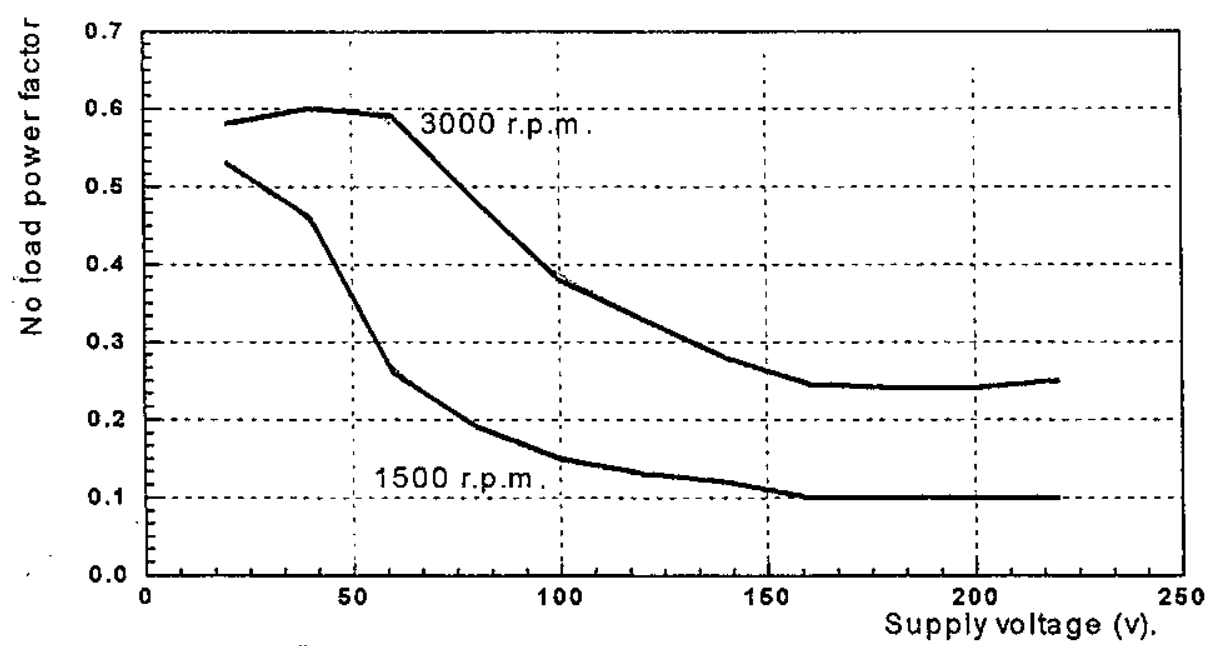

F Fig.(10).No load power factor versus supply voltage.

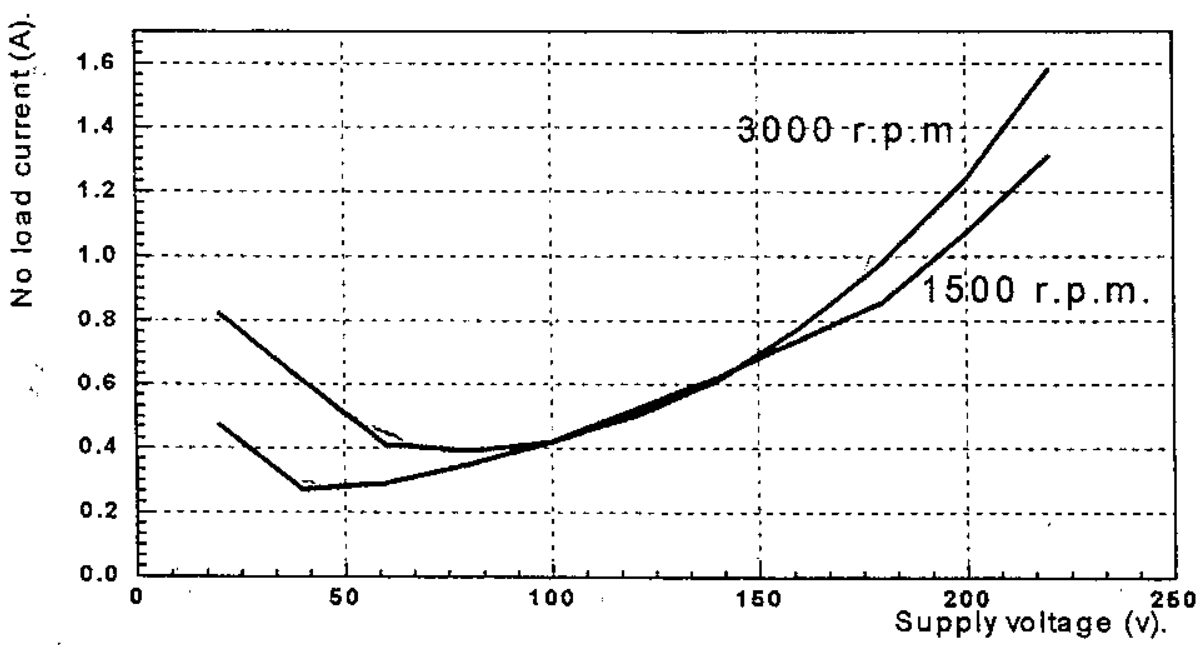

Fig.(11).No load current versus supply voltage. 


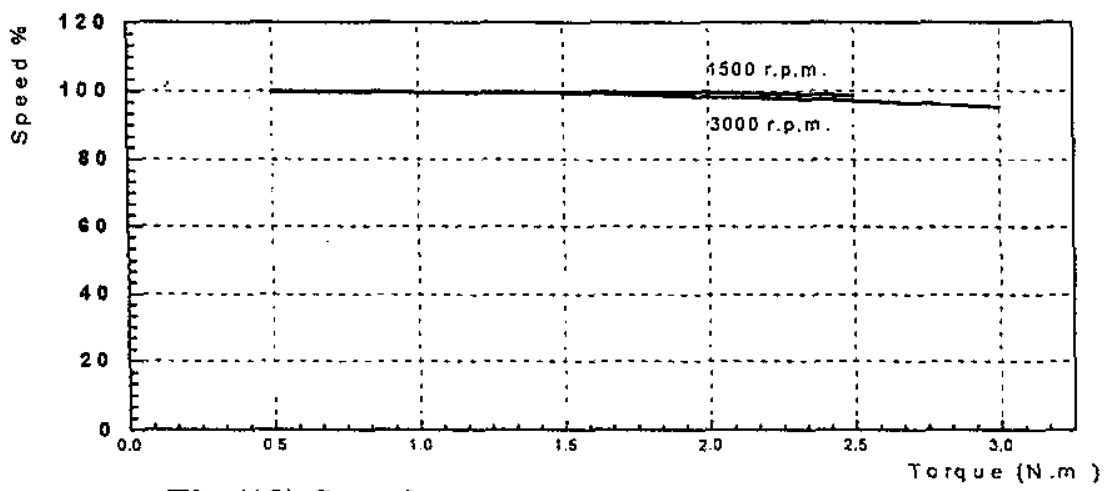

Fig.(12).Speed versus output torque.

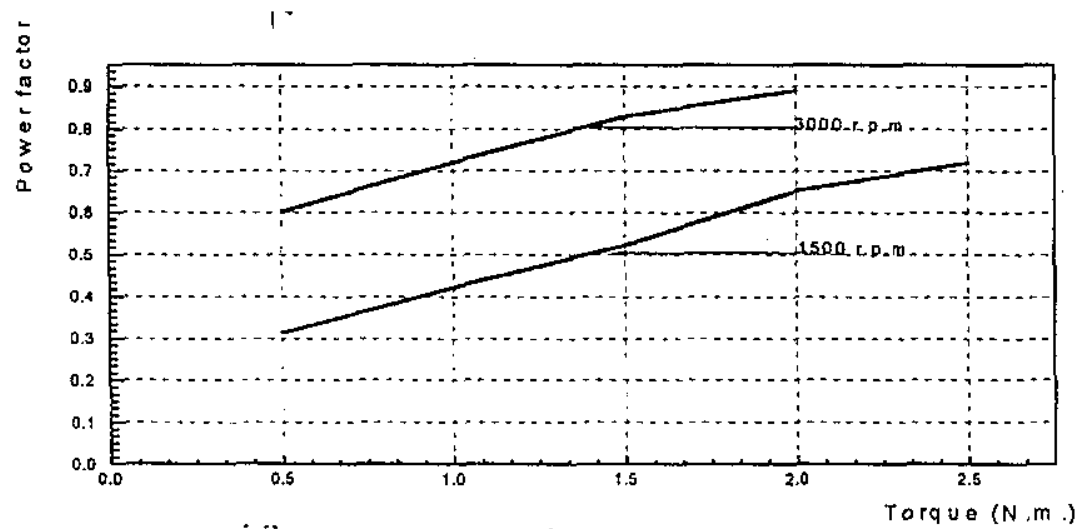

Fig.(13).Load power factor versus output torque.

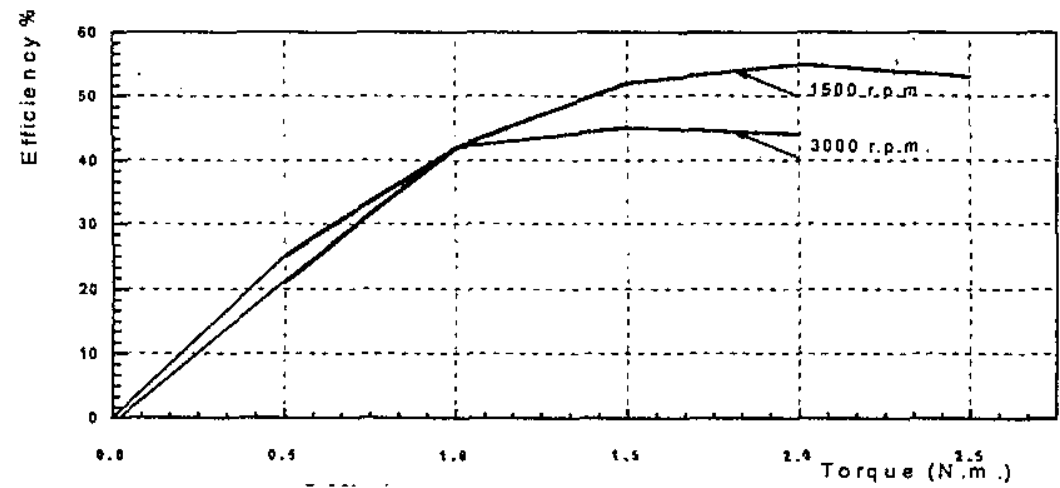

Fig.(14).Efficiency versus output torque.

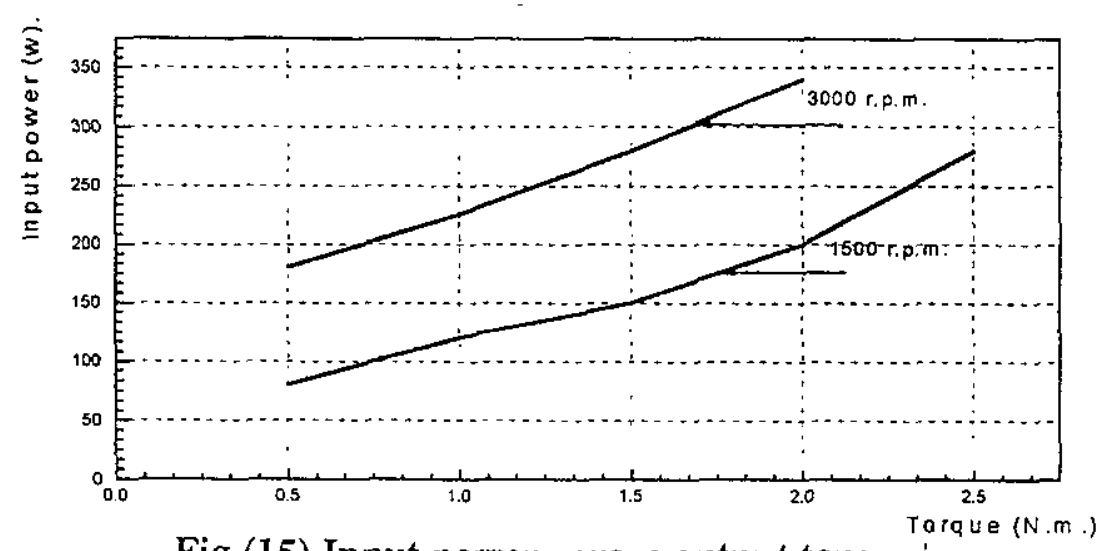

Fig (15) Input power versus output torque'. 
فلسفة جليدة لتمدليل قيمة القطب

فى المحركات التأثيرية ثلاثية الأوجه

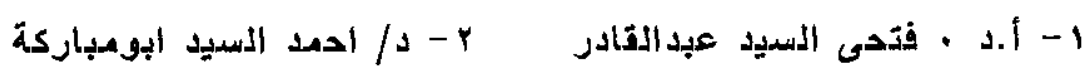

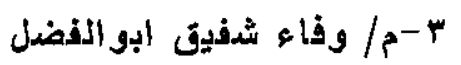

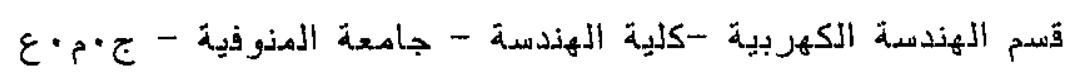

ملفخص البحث

تعتبر المحركـات التأثيريسة ثلاثيـة الأوجـه مـن أفضـل أنــواع المحركــات الكهربية · حيث نكون أعلى كفاعة و أقل تكلفة · إلا أن العيب الرئيسى لهـا

$$
\text { هو صعوبة التحكم فى سرعنها }
$$

يتناول هذا البحث طريقة للتحكم فىى السـر عة بو اسـطة تعديـل عددد وقيمسة الأقطاب حيث ذكرت الأبحاث السابقة أنه يمكن الحصول على هـذا التحكم بعدة طرق إلا أنه بتطبيق هذه الطرق ثبت انها لاتقوم بالتحكم المطلوب . ودم فى البحث در اسة أسباب عدم الحصول علىى هـذا التحكم بهذه الطـرق ونم الذوصل الى الشروط اللازم تدقيقها لكى يذم التحكم فى السرعة بنعديل عدد وقيمة الأقطاب - مداب 